\title{
SCENARIOS OF OPTIMAL CONTROL OF TRANSREGIONAL MIGRATION PROCESSES UNDER RISK
}

\author{
V. V. Akimenko, A. G. Nakonechnyi, and S. D. Voloshchuk
}

UDC $519.7(075.8)$

\begin{abstract}
Scenarios of migration are considered as applied to the problem of optimal control of transregional migration with zero risk of overpopulation or insufficient population and migration with obtaining the minimum of the objective functional and minimizing risk. Numerical algorithms for the scenarios considered are developed. The algorithms are implemented in an applied geoinformation decision-support system for control of transregional migration of population.
\end{abstract}

Keywords: optimal control, discrete optimization, migration, utility function, dynamic system, demographic risk.

\section{INTRODUCTION}

Scenarios of optimal control of transregional migration processes that are presented in this paper are specific to the optimal control problem $A 2$ from [1] and are based on models of the two-level hierarchical organizational system [2-4] of optimal control for linear systems of ordinary differential equations [5-7] and models of the utility function for demographic processes [8]. The general algorithm proposed in [1] for solution of this problem in the most general statement leads to a time-consuming discrete optimization problem whose solution lies in finding the global extremum of a function of a large number $N_{\text {tot }}$ (that amounts to about several hundreds) of variables. For practical operation of geoinformation application systems in real-time, individual scenarios should be considered that, on the one hand, form a modified class of subtasks with additional constraints and, on the other hand, require the development of more efficient methods (algorithms) of searching for optimal solutions. In this work, we present (1) the scenario of absence of demographic risk at the initial moment of time with preservation of this condition over the whole territory at subsequent moments of time with the minimization of the objective functional of population distribution over given domains; (2) the scenario of a given population distribution over chosen regions (oblasts) of the domain being considered at the initial and subsequent moments of time with the minimization of the objective functional of total demographic risk in the other regions of the oblast. New algorithms are developed for the directed search for solution as applied to these scenarios (optimal control problems formulated in a new form) on the basis of analyzing gradients of objective functionals, taking into account the specificity of the domain of minimization, and using the methodology of sequential analysis of variants [9]. In this article, the results of functioning of the algorithms implemented in a geoinformation support system of making decisions on optimal control of transregional migration processes under risk are illustrated by an example of socioeconomic and ecological data for Ukraine over 2001-2004.

\section{STATEMENT OF THE GENERAL PROBLEM OF OPTIMAL CONTROL OF MIGRATION PROCESSES UNDER RISK}

Let us consider an optimal control model for the speed problem specified on the basis of a linear system of ordinary differential equations. We introduce the dependence of the parameters of the system on time $t$, on the identification parameter

Taras Shevchenko University, Kiev, Ukraine. Translated from Kibernetika i Sistemnyi Analiz, No. 1, pp. 16-33, January-February 2007. Original article submitted January 4, 2006. 
of a region of the society (state) $\omega \in \Omega$ (the number of the region can be used as such a parameter) for a finite discrete set that specifies the area of investigation $\Omega$, on the number of a social group $k \in K_{0} \subset N$, and on socioeconomic and ecological parameters $x$ of the system. Within the framework of a two-level hierarchical system Center $C_{0}$, a bounded collection of dynamic control parameters is used, namely, a vector function $g(t)=\left(g_{1}(t), \ldots, g_{n}(t)\right)$ for the determination of dynamic controlled socioeconomic parameters (trajectories of a process $\left.x(t, \omega, k, g)=\left(x_{1}(t, \omega, k, g), \ldots, x_{n}(t, \omega, k, g)\right)\right)$ with a view to coordinating social groups $C_{k}$. To form a hypothesis on the behavior of a group $C_{k}$, a model of the scalar utility function $u(t, x, \omega, k)$ is used that is the life quality index of the social group in a region $\omega$ at a moment of time $t$ with given parameters $x=x(t, \omega, k, g)$. It functions as a binary relation and determines the advantage (equivalence) of the first collection of parameters $(t, x, \omega, k)^{(1)}$ over the second collection $(t, x, \omega, k)^{(2)}$ if we have $u(t, x, \omega, k)^{(1)} \geq u(t, x, \omega, k)^{(2)}$. The hypothesis of optimal behavior of the group $C_{k}$ at a fixed moment of time $t$ is specified with the help of an empirical function of migration balance $M=M(u(t, x, \omega, k, g))$ that represents the difference between the numbers of citizens who arrive at and depart from the region during a definite time period. We introduce the following designations: $D(t, x, \omega, k, g)$ denotes the population size, $\widetilde{D}\left(T_{0}, \omega, k\right)$ is the desirable population size in regions $\omega \in \Omega_{1}: \Omega_{1} \neq \varnothing, \Omega_{2}=\Omega \backslash \Omega_{1} \neq \varnothing$ that is established by the Center $C_{0}, D_{0}$ is the initial population size, $R$ is the balance between birth and death rates during a definite time period (a prescribed step function), $M_{E}$ is the balance of abroad migration during a definite time period (a prescribed function), $\chi(x)$ is the Heaviside function; coefficients $\alpha_{0}$ and $\alpha_{k}$, an odd parameter $q \in N$, a parameter $j_{0}$, and a weight coefficient $\gamma_{l}(k) \neq 0$ are parameters specified by experts (when $\gamma_{l}>0$, the effect of the parameter $x_{l}$ on the migration process is positive and on the emigration process is negative, and when $\gamma_{l}<0$, this effect is inverse); $\bar{x}_{l}$ and $\hat{x}_{l}$ are expert estimates of the "inertness" of the $k$ th social group with respect to the parameter $x_{l} ; A$ is a given linear continuous operator $\left(R^{n} \rightarrow R^{n}\right), r(t, \omega, k, g)$ is the value of the risk of overpopulation or insufficient population that belongs to a social group $C_{k}$ and lives in the oblast $\omega$ at the moment $t, \breve{D}(t, \omega, k)$ and $\widehat{D}(t, \omega, k)$ are given threshold values of the population size, and $\varepsilon>0$ is an error. We formulate the problem of optimal control [1] in the form

$$
\begin{aligned}
& G^{*}=\operatorname{Arg} \min _{g \in G}\left(J_{1}\left(T_{0}, k, g\right)+J_{2}\left(T_{0}, k, g\right)\right), \\
& J_{1}\left(T_{0}, k, g\right)=\sum_{\omega \in \Omega_{1}} \lambda_{1}(\omega)\left[\widetilde{D}\left(T_{0}, \omega, k\right)-D\left(T_{0}, x, \omega, k, g\right)\right]^{2}, \\
& J_{2}\left(T_{0}, k, g\right)=\sum_{\omega \in \Omega} \lambda_{2}(\omega) r\left(T_{0}, \omega, k, g\right), \\
& \dot{D}(t, x, \omega, k, g)=M(u(t, x, \omega, k, g))+M_{E}(t, \omega, k)+R(t, \omega, k), \\
& D\left(t_{0}, \omega, k\right)=D_{0}(\omega, k), \\
& \dot{x}(t, \omega, k)=A g, \quad x\left(t_{0}, \omega, k\right)=x^{0}(\omega, k), \\
& x \in X(t) \subset R^{n}, \quad X(t)=X_{1}(t) \times \ldots \times X_{n}(t), \\
& g \in G \subset R^{n}, \quad g^{*} \in G^{*} \subset G, \quad t \in\left[t_{0}, T_{0}\right], \omega \in \Omega, \\
& M(u(t, x, \omega, k, g))=\alpha_{0}+\sum_{j=1}^{j_{0}} \alpha_{j}(u(t, x, \omega, k, g))^{2 j-1}, \alpha_{j} \geq 0 \quad\left(j=\overline{1, j_{0}}\right), \\
& u(t, x, \omega, k)=\sum_{l=1}^{n} \gamma_{l}(k)\left(\left(x_{l}(t, \omega, k, g)-\breve{x}_{l}(k)\right) \chi\left(\breve{x}_{l}(k)-x_{l}(t, \omega, k, g)\right)\right. \\
& \left.+\left(x_{l}(t, \omega, k, g)-\widehat{x}_{l}(k)\right) \chi\left(x_{l}(t, \omega, k, g)-\widehat{x}_{l}(k)\right)\right)^{q}, \\
& r(t, x, \omega, k, g)=\frac{1}{t-t_{0}} \int_{t_{0}}^{t}\{(\breve{D}(t, \omega, k)-D(M(t, x, \omega, k, g))) \chi(\breve{D}-D(M(t, x, \omega, k, g))) \\
& +(D(M(t, \omega, k, g))-\widehat{D}(t, \omega, k)) \chi(D(M(t, \omega, k, g))-\widehat{D}(t, \omega, k))\}^{2} d t+r_{0}(\omega, k),
\end{aligned}
$$




$$
\begin{gathered}
\sum_{\omega \in \Omega} M(u(t, x, \omega, k, g))=0, \quad \hat{G}_{0}^{*}=\operatorname{Arg} \min _{\hat{g}^{*} \in \hat{G}^{*}}\left(T\left(k, \hat{g}^{*}\right)-t_{0}\right), \\
\quad\left|J_{1}\left(T, k, \hat{g}^{*}\right)-J_{1}\left(T_{0}, k, g^{*}\right)\right| \leq \varepsilon, \quad T \in\left[t_{0}, T_{0}\right], \\
\hat{G}^{*}=\left\{\hat{g}^{*}(t) \mid \hat{g}^{*}(t)=\left\{\begin{array}{cc}
g^{*}(t), & t \in\left[t_{0}, T\right], \\
0, & t \in\left(T, T_{0}\right],
\end{array} g^{*}(t) \in G^{*}\right\},\right.
\end{gathered}
$$

where $\lambda_{1}(\omega)$ and $\lambda_{2}(\omega)$ are positive dimensionless weight coefficients. The solution of problem (1) is a set of optimal controls $\hat{g}^{(0)^{*}} \in \hat{G}_{0}^{*} \subset G \subset R^{n}$. The conditions of Theorem 2 from [1] are considered to be true since $g$ and its derivative $g_{t}$ are bounded within $L_{2}\left(\left[t_{0}, T_{0}\right]\right)$, whence the compactness of the set $G$ follows.

\section{SCENARIO OF AN OPTIMAL CONTROL WITH ZERO RISK}

For problem (1), we consider the case when $A=E$ is a unit operator. This scenario is based on the following additional constraints on the parameters of the problem:

$$
\begin{gathered}
r_{0}(\omega, k)=0, \\
W(t, \omega, k)=(D(M(t, x, \omega, k, g))-\breve{D}(t, \omega, k)) \chi(\breve{D}-D(M(t, x, \omega, k, g))) \\
+(D(M(t, x, \omega, k, g))-\widehat{D}(t, \omega, k)) \chi(D(M(t, x, \omega, k, g))-\widehat{D}(t, \omega, k))=0, \\
t \in\left[t_{0}, T_{0}\right], \omega \in \Omega, \quad k \in K_{0}, \quad g \in G .
\end{gathered}
$$

Constraints (2) require that risk be equal to zero at the initial moment of time and that these conditions be preserved during each subsequent moment of time over the entire given territory. Taking into account the nonnegativity of the functionals $J_{1}$ and $J_{2}$, condition (2) practically means that the Center $C_{0}$ considers a scenario of development of the migration process for the case when the absence of demographic risk is more preferable (the functional $J_{2}$ ) than the attainment of an optimal distribution of social groups among regions (the functional $J_{1}$ ), i.e., the weight coefficient $\lambda_{1}<<\lambda_{2}$. Condition (2) forms a new domain of admissible system trajectories and, accordingly, a domain of admissible controls that is determined by the following inequalities:

$$
\begin{gathered}
P_{1}(t, \omega, k) \leq \int_{t_{0}}^{t} \sum_{j=1}^{j_{0}} \alpha_{j}\left(\sum _ { l = 1 } ^ { n } \gamma _ { l } ( k ) \left(( x _ { l } ( \tau , \omega , k , g ) - \breve { x } _ { l } ( k ) ) \chi \left(\breve{x}_{l}(k)\right.\right.\right. \\
\left.-x_{l}(\tau, \omega, k, g)\right)+\left(x_{l}(\tau, \omega, k, g)\right. \\
\left.\left.\left.-\widehat{x}_{l}(k)\right) \chi\left(x_{l}(\tau, \omega, k, g)-\widehat{x}_{l}(k)\right)\right)^{q}\right)^{2 j-1} d \tau \leq P_{2}(t, \omega, k), \\
P_{1}(t, \omega, k)=\breve{D}(t, \omega, k)-D_{0}\left(t_{0}, \omega, k\right)-\alpha_{0}\left(t-t_{0}\right) \\
-\int_{t_{0}}^{t}\left(M_{E}(\tau, \omega, k)+R(\tau, \omega, k)\right) d \tau, \\
P_{2}(t, \omega, k)=\widehat{D}(t, \omega, k)-D_{0}\left(t_{0}, \omega, k\right)-\alpha_{0}\left(t-t_{0}\right)-\int_{t_{0}}^{t}\left(M_{E}(\tau, \omega, k)+R(\tau, \omega, k)\right) d \tau .
\end{gathered}
$$

Since control is considered on the class of bounded step functions (the compactness of the set $G$ ) [1], and condition (3) determines a compact set of optimal controls $g \in \widetilde{G} \subset G$. Problem (1), (2) is correctly stated. To construct a numerical 
algorithm, we consider the covering of the segment $\left[t_{0}, T_{0}\right]$ by a finite set of identical segments $\left[t_{p-1}, t_{p}\right], \tau_{0}=t_{p}-t_{p-1}$, $p=\overline{1, P_{0}}, t_{P_{0}}=T_{0}$. Control is considered on the class of bounded step functions

$$
\begin{gathered}
g^{(0)}(t, \omega, k)=a_{0}(t, \omega, k)=\sum_{p=1}^{P_{0}}\left(\chi\left(t-t_{p-1}\right)-\chi\left(t-t_{p}\right)\right) g_{p}(\omega, k), \\
\breve{L}_{l p}(\omega, k) \leq g_{l p}(\omega, k) \leq \widehat{L}_{l p}(\omega, k),
\end{gathered}
$$

where $\breve{L}_{l p}(\omega, k) \leq 0 \leq \widehat{L}_{l p}(\omega, k)$ are constants specified by experts for $p=\overline{1, P_{0}}, l=\overline{1, n}, \omega \in \Omega, k \in K_{0}$. The existence at least one optimal control satisfying condition (2) over the class of step functions follows from the form of constraints (4) for the compact set $G$ and zero initial conditions for risks. Speed problem (1), (2) can be formulated in terms of discrete optimization theory [9]. We represent the functional of the population size in the additive form

$$
\begin{gathered}
D\left(t_{p}, \omega, k, g\right)=D_{0}(\omega, k)+\tau_{0}\left(P \alpha_{0}+\sum_{p=1}^{P}\left(M_{E}\left(t_{p}, \omega, k\right)+R\left(t_{p}, \omega, k\right)\right)\right) \\
+\sum_{p=1}^{P} \sum_{j=1}^{j_{0}} \alpha_{j} \int_{t_{p-1}}^{t_{p}}\left(\sum _ { l = 1 } ^ { n } \gamma _ { l } ( k ) \left(\left(\left(\tau-t_{p-1}\right) g_{l p}+x_{l}\left(t_{p-1}, \omega, k, g_{l p-1}\right)-\breve{x}_{l}(k)\right)\right.\right. \\
\times \chi\left(\left(\tau-t_{p-1}\right) g_{l p}+x_{l}\left(t_{p-1}, \omega, k, g_{l p-1}\right)-\breve{x}_{l}(k)\right) \\
+\left(\left(\tau-t_{p-1}\right) g_{l p}+x_{l}\left(t_{p-1}, \omega, k, g_{l p-1}\right)-\widehat{x}_{l}(k)\right) \\
\left.\left.\times \chi\left(\left(\tau-t_{p-1}\right) g_{l p}+x_{l}\left(t_{p-1}, \omega, k, g_{l p-1}\right)-\widehat{x}_{l}(k)\right)\right)^{q}\right)^{2 j-1} d \tau \\
x_{l}\left(t_{p}, \omega, k, g_{l p}\right)=\tau_{0} \sum_{i=1}^{p} g_{l i}(\omega, k)+x_{l}^{0}(\omega, k) \\
g_{0}(\omega, k)=\left(g_{10}(\omega, k), \ldots, g_{n 0}(\omega, k)\right)=0 .
\end{gathered}
$$

By analogy with the methodology of sequential analysis of variants, for each segment $\left[t_{p-1}, t_{p}\right]\left(p=\overline{1, P_{0}}\right)$, we specify a collection of control parameters $g \in \widetilde{G} \subset G$ that provide the minimum of the functional

$$
g_{p}^{*}(\omega, k)=\left(g_{1 p}^{*}(\omega, k), \ldots, g_{n p}^{*}(\omega, k)\right)=\arg \inf _{g_{p} \in \widetilde{G}}\left(J_{1}\left(t_{p}, k, g_{p}\right)\right)
$$

successively for each $p=1,2, \ldots$. Computations come to an end after the attainment of the minimum of functional (6) with a given accuracy $\varepsilon>0$ when $p=p^{*}$ or a final moment of time when $p=P_{0}$. Using the theorem on the differentiation of an integral with respect to a parameter, we determine the gradient of the functional $J_{1}$ with the help of the function of the population size from functional (5) in the segment $\left[t_{0}, t_{p}\right]$ as follows:

$$
\begin{gathered}
\frac{\partial J_{1}\left(t_{p}, k, g\right)}{\partial g_{l^{*} p}(\omega, k)}=2 \lambda_{1}(\omega)\left[\widetilde{D}\left(T_{0}, \omega, k\right)-D\left(t_{p}, x, \omega, k, g\right)\right] \frac{\partial D\left(t_{p}, x, \omega, k, g\right)}{\partial g_{l^{*} p}(\omega, k)}, \\
\frac{\partial D}{\partial g_{l^{*} p}(\omega, k)}=\gamma_{l^{*}}(k) \sum_{j=1}^{j_{0}} \alpha_{j} \int_{t_{p-1}}^{t_{p}}\left(\sum _ { l = 1 } ^ { n } \gamma _ { l } ( k ) \left(\left(\left(\tau-t_{p-1}\right) g_{l p}+x_{l}\left(t_{p-1}, \omega, k, g_{l p-1}\right)\right.\right.\right. \\
\left.-\breve{x}_{l}(k)\right) \chi\left(\left(\tau-t_{p-1}\right) g_{l p}+x_{l}\left(t_{p-1}, \omega, k, g_{l p-1}\right)-\breve{x}_{l}(k)\right)+\left(\left(\tau-t_{p-1}\right) g_{l p}+x_{l}\left(t_{p-1}, \omega, k, g_{l p-1}\right)-\widehat{x}_{l}(k)\right)
\end{gathered}
$$




$$
\begin{gathered}
\left.\left.\times \chi\left(\left(\tau-t_{p-1}\right) g_{l p}+x_{l}\left(t_{p-1}, \omega, k, g_{l p-1}\right)-\widehat{x}_{l}(k)\right)\right)^{q}\right)^{2 j-2} \\
\times(2 j-1) q\left(( ( \tau - t _ { p - 1 } ) g _ { l ^ { * } p } + x _ { l ^ { * } } ( t _ { p - 1 } , \omega , k , g _ { l ^ { * } p - 1 } ) - \breve { x } _ { l ^ { * } } ( k ) ) \chi \left(\left(\tau-t_{p-1}\right) g_{l^{*} p}\right.\right. \\
\left.+x_{l^{*}}\left(t_{p-1}, \omega, k, g_{l^{*} p-1}\right)-\breve{x}_{l^{*}}(k)\right)+\left(\left(\tau-t_{p-1}\right) g_{l^{*} p}+x_{l^{*}}\left(t_{p-1}, \omega, k, g_{l^{*} p-1}\right)\right. \\
\left.\left.-\widehat{x}_{l^{*}}(k)\right) \chi\left(\left(\tau-t_{p-1}\right) g_{l^{*} p}+x_{l^{*}}\left(t_{p-1}, \omega, k, g_{l^{*} p^{*}-1}\right)-\widehat{x}_{l^{*}}(k)\right)\right)^{q-1} \\
\times\left(\tau-t_{p-1}\right)\left(\chi\left(\left(\tau-t_{p-1}\right) g_{l^{*} p}+x_{l^{*}}\left(t_{p-1}, \omega, k, g_{l^{*} p-1}\right)-\bar{x}_{l^{*}}(k)\right)\right. \\
\left.\left.+\chi\left(\left(\tau-t_{p-1}\right) g_{l^{*} p}+x_{l^{*}}\left(t_{p-1}, \omega, k, g_{l^{*} p-1}\right)-\widehat{x}_{l^{*}}(k)\right)\right)\right) d \tau .
\end{gathered}
$$

Since the exponents $(2 j-2)$ and $(q-1)$ are even and coefficients $\alpha_{j} \geq 0\left(j=\overline{1, j_{0}}\right)$ and $\tau \geq t_{p-1}$, the subintegral function in formula (8) is nonnegative. It follows from formulas (7) and (8) that the sign of the $l^{*}$-component of the gradient of the functional $J_{1}$ is determined by the sign of $\left(\widetilde{D}\left(T_{0}, \omega, k\right)-D\left(t_{p}, x, \omega, k, g\right)\right)$ and the sign of the parameter $\gamma_{l^{*}}(k)$. In fact, this means that the optimal values of the $l^{*}$-component of the system trajectory $x_{l^{*}}\left(t_{p}\right)$ are in the segment connecting the initial value of the system trajectory with the projection of a given point onto the boundary of the compact domain determined by the constraints of problem (1) and inequalities (3). But the components of the gradient of the functional can assume zero values not only in the domain of the minimum of the functional but also in the domain of inertness of the utility function (the presence of the Heaviside function under the integration sign). Therefore, along with formulas (7) and (8), one should also take into account the smoothness properties of the function $D\left(t_{p}, x, \omega, k, g\right)$ that enters in the expressions for both functionals. Let us consider a numerical algorithm for solution of problem (1). We fix $k \in K_{0}$ (the $C_{k}$ th social group).

1. Specify the initial parameters and characteristic constants of problem (1).

2. Specify the current step with respect to time $p \geq 1$ and determine a moment of time $t_{p}=t_{p-1}+\tau_{0}$.

3. Fix the sequent region $\omega \in \Omega_{1}$. For this region, determine the boundary value of the population size $D_{r}\left(t_{p}, \omega, k\right)$ for zero risk as follows:

$$
D_{r}\left(t_{p}, \omega, k\right)= \begin{cases}\widetilde{D}\left(T_{0}, \omega, k\right) & \text { if } \quad \widetilde{D}\left(T_{0}, \omega, k\right) \in\left[\breve{D}\left(t_{p}, \omega, k\right), \widehat{D}\left(t_{p}, \omega, k\right)\right], \\ \widetilde{D}\left(t_{p}, \omega, k\right) & \text { if } \quad \widetilde{D}\left(T_{0}, \omega, k\right)>\widehat{D}\left(t_{p}, \omega, k\right), \\ \widetilde{D}\left(t_{p}, \omega, k\right) & \text { if } \quad \widetilde{D}\left(T_{0}, \omega, k\right)<\breve{D}\left(t_{p}, \omega, k\right) .\end{cases}
$$

4. According to formulas (7) and (8), determine the initial value of the auxiliary control parameter $\Delta_{l p}(\omega, k), l=\overline{1, n}$, as follows:

$$
\Delta_{l p}(\omega, k)=\left\{\begin{array}{lll}
\widehat{L}_{l p}(\omega, k) / N_{\gamma} & \text { if } \quad\left(\gamma_{l}(k)\left(D\left(t_{p-1}, x, \omega, k, g\right)-D_{r}\left(t_{p}, \omega, k\right)\right)\right)<0 \\
\breve{L}_{l p}(\omega, k) / N_{\gamma} & \text { if } \quad\left(\gamma_{l}(k)\left(D\left(t_{p-1}, x, \omega, k, g\right)-D_{r}\left(t_{p}, \omega, k\right)\right)\right)>0 \\
0 & \text { if } \quad\left(\gamma_{l}(k)\left(D\left(t_{p-1}, x, \omega, k, g\right)-D_{r}\left(t_{p}, \omega, k\right)\right)\right)=0
\end{array}\right.
$$

where $N_{\gamma}$ is the initial value of the number of steps along the segment connecting the initial point of the system trajectory and the boundary point determined by the boundary value of a compact set $G \subset R^{n}$.

In this case, the use of the gradient of a functional according to formulas (7) and (8) is, on the one hand, sufficiently time-consuming for the numerical algorithm and, on the other hand, one should realize additional checks and execute procedures similar to (9) in the cases when all the components of the gradient of the functional equal zeros and when the system trajectory has not yet attained the minimum point (the domain of inertness of the utility function). However, formulas (5), (7), and (8) imply that the maximal value of the population size $D_{\max }\left(t_{p}, \omega\right)$ is attained when the system moves in the direction of increasing the values of stimulators $g_{l p}(\omega, k)=\widehat{L}_{l p}(\omega, k)$ (when $\left.\gamma_{l}>0\right)$ to their maximum and decreasing the values of destimulators of a social system $g_{l p}(\omega, k)=\breve{L}_{l p}(\omega, k)$ (when $\left.\gamma_{l}<0\right)$ to their minimum. The minimal value of the population size $D_{\min }\left(t_{p}, \omega\right)$ is similarly attained when the system moves in the direction of decreasing the values of 
stimulators $g_{l p}(\omega, k)=\widehat{L}_{l p}(\omega, k)$ to their minimum (when $\left.\gamma_{l}>0\right)$ and increasing the values of the destimulators of the social system $g_{l p}(\omega, k)=\breve{L}_{l p}(\omega, k)$ to their maximum (when $\left.\gamma_{l}<0\right)$. It is obvious that the population size assumes a value that minimizes the functional $J_{1}$ when $J_{2}=0$, within the internal domain of the set $\widetilde{G}$ when $D_{\min }\left(t_{p}, \omega\right)<D_{r}\left(t_{p}, \omega\right)<$ $D_{\max }\left(t_{p}, \omega\right)$, or on the boundary of the set $\widetilde{G}$ when $D_{r}\left(t_{p}, \omega\right)>D_{\max }\left(t_{p}, \omega\right)$ or $D_{r}\left(t_{p}, \omega\right)<D_{\min }\left(t_{p}, \omega\right)$. In the latter case, optimal values of control parameters are located on the boundary of the domain $g_{l p}^{*}(\omega, k)=\widehat{L}_{l p}(\omega, k)\left(\right.$ when $\left.\gamma_{l}>0\right)$, $g_{l p}^{*}(\omega, k)=\breve{L}_{l p}(\omega, k)\left(\right.$ when $\left.\gamma_{l}<0\right)$ or $g_{l p}^{*}(\omega, k)=\breve{L}_{l p}(\omega, k)\left(\right.$ when $\left.\gamma_{l}>0\right)$, and $g_{l p}^{*}(\omega, k)=\widehat{L}_{l p}(\omega, k)\left(\right.$ when $\left.\gamma_{l}<0\right)$. It is also obvious that an optimal control within the internal domain $\widetilde{G}$ can be not unique. Then a unique solution is chosen by means of an additional requirement of the minimization of the computational complexity of the algorithm, for example, by the construction of a minimizing sequence by formulas (9) and (10).

5. Compute the value $(s)$ of the control parameter

$$
g_{l p}^{(s)}=g_{l p}^{(s-1)}+\Pi\left(\alpha^{(s)}(\omega, k) \Delta_{l p}(\omega, k)\right), g_{l p}^{(0)}=0, \alpha^{(1)}(\omega, k)=1 \quad(s \geq 1),
$$

where $0<\alpha^{(s)}(\omega, k)<1$ is an auxiliary scalar parameter and $\Pi$ is the operator of projection onto the boundary of a domain $g_{l p}^{(s)}(\omega, k) \in\left[\breve{L}_{l p}(\omega, k), \widehat{L}_{l p}(\omega, k)\right]$. Compute the coordinates of the $(s)$-element of the minimizing sequence, i.e., the system trajectory

$$
x_{l}^{(s)}\left(t_{p}, \omega, k, g_{l p}\right)=\tau_{0} g_{l p}^{(s)}(\omega, k)+x_{l}\left(t_{p-1}, \omega, k, g_{l p}\right), \quad \omega \in \Omega_{1}, \quad l=\overline{1, n} \quad(s \geq 1) .
$$

Compute the values of functions $u\left(t_{p}, x^{(s)}, \omega, k\right), M\left(u\left(t_{p}, x^{(s)}, \omega, k, g\right)\right)$, and $D\left(t_{p}, x^{(s)}, \omega, k, g\right)$.

6. Check the following convergence condition with the help of the minimum residual condition (the proximity of components of the gradient of the functional to zero) with a given error $\varepsilon_{1}$ :

$$
\left(D_{r}\left(t_{p}, \omega, k\right)-D\left(t_{p}, x^{(s)}, \omega, k, g^{(s)}\right)\right)^{2} \leq \varepsilon_{1}\left\|\widetilde{D}\left(T_{0}\right)\right\|_{C\left(\Omega \times K_{0}\right)}^{2}
$$

If condition (12) is true, then go to item 7. Otherwise, check the following conditions of the monotony of the residual:

$$
\begin{aligned}
& \left(D_{r}\left(t_{p}, \omega, k\right)-D\left(t_{p}, x^{(s)}, \omega, k, g^{(s)}\right)\right)^{2}<\left(D_{r}\left(t_{p}, \omega, k\right)-D\left(t_{p}, x^{(s-1)}, \omega, k, g^{(s-1)}\right)\right)^{2}, \\
& \left(D_{r}\left(t_{p}, \omega, k\right)-D\left(t_{p}, x^{(s)}, \omega, k, g^{(s)}\right)\right) \times\left(D_{r}\left(t_{p}, \omega, k\right)-D\left(t_{p}, x^{(s-1)}, \omega, k, g^{(s-1)}\right)\right)>0 .
\end{aligned}
$$

If conditions (13) and (13a) are true, increase $s$ by unity, assume that $\alpha^{(s)}=\alpha^{(s-1)}$, and go to the beginning of item 5 to compute the system trajectory at the new step of iterations. Otherwise, decrease the value of $\alpha^{(s)}$ by a factor of two and check the following condition of attainability of the boundary of resources in the region: $\alpha^{(s)}<\varepsilon_{2}$. If this condition is true, then go to item 7 and, otherwise, go to the beginning of item 5 and compute the $(s)$-values of the control parameter and system trajectory once again.

7. If the set $\Omega_{1}$ is not exhausted, then go to item 3 and, otherwise, go to item 8 .

8. Check the following condition of violation of the migration balance as a result of a change in control parameters in regions $\omega \in \Omega_{1}$ :

$$
\Delta M_{\Omega_{1}}=\sum_{\omega \in \Omega_{1}} M\left(t_{p}, x, \omega, k, g\right)-\sum_{\omega \in \Omega_{1}} M\left(t_{p-1}, x, \omega, k, g\right)=0 .
$$

If condition (14) is true, then the migratory balance is preserved in regions $\omega \in \Omega_{1}$ and this implies the preservation of the balance in regions $\omega \in \Omega_{2}$. Since the condition of absence of risk is true at the moment $t_{p-1}$, put the values of $g_{l p}^{(s)}(\omega, k)=0$ for control parameters for regions $\omega \in \Omega_{2}$ and go to item 11.

Otherwise, for each region $\omega \in \Omega_{2}$, determine maximally admissible values of control parameters as follows:

$$
g_{l p}(\omega, k)=\left\{\begin{array}{lll}
\widehat{L}_{l p}(\omega, k) & \text { if } \quad\left(\gamma_{l}(k) \Delta M_{\Omega_{1}}\right)<0, \\
\breve{L}_{l p}(\omega, k) & \text { if } \quad\left(\gamma_{l}(k) \Delta M_{\Omega_{1}}\right)>0 .
\end{array}\right.
$$


Since the migration balance equation has been true at the previous moment of time $t_{p-1}$, based on the analysis of the change in the direction of the migration process (obtained as a result of solution of the optimization problem for the domain $\Omega_{1}$ ), the greatest possible social and economic resources of the domain $\Omega_{2}$ at the current step are determined that are necessary for the compensation of the arising disbalance. For greatest possible controls (15), compute the system trajectory $x_{l}^{l}\left(t_{p}, \omega, k, g_{l p}\right)$ by formula (11) and the values of functions $u\left(t_{p}, x^{(l)}, \omega, k\right), M\left(u\left(t_{p}, x^{(l)}, \omega, k, g\right)\right)$, and $D\left(t_{p}, x^{(l)}, \omega, k, g\right)$. Taking into account risk, correct the value of the population size resource as follows:

$$
D_{r}\left(t_{p}, x^{(l)}, \omega, k, g\right)=\left\{\begin{array}{llc}
D\left(t_{p}, x^{(l)}, \omega, k, g\right) & \text { if } & D\left(t_{p}, x^{(l)}, \omega, k, g\right) \in\left[\breve{D}\left(t_{p}, \omega, k\right), \widehat{D}\left(t_{p}, \omega, k\right)\right], \\
\bar{D}\left(t_{p}, \omega, k\right) & \text { if } & D\left(t_{p}, x^{(l)}, \omega, k, g\right)>\bar{D}\left(t_{p}, \omega, k\right), \\
\bar{D}\left(t_{p}, \omega, k\right) & \text { if } & D\left(t_{p}, x^{(l)}, \omega, k, g\right)<\breve{D}\left(t_{p}, \omega, k\right) .
\end{array}\right.
$$

Determine the corresponding values of $M_{r}$ by the formula

$$
M_{r}\left(t_{p}, x^{(l)}, \omega, k, g\right)=\frac{1}{\tau_{0}}\left(D_{r}\left(t_{p}, x^{(l)}, \omega, k, g\right)-D\left(t_{p-1}, x, \omega, k, g\right)\right)-\left(M_{E}\left(t_{p}, \omega, k\right)+R\left(t_{p}, \omega, k\right)\right) .
$$

By virtue of expressions (15) and (16), the following inequality is true:

$$
\sum_{\omega \in \Omega_{2}}\left(M_{r}\left(t_{p}, x^{(l)}, \omega, k, g\right)-M\left(t_{p-1}, \omega, k\right)\right) \sum_{\omega \in \Omega_{1}}\left(M\left(t_{p}, x^{(s)}, \omega, k, g\right)-M\left(t_{p-1}, \omega, k\right)\right) \leq 0 .
$$

9. If we have

$$
\left|\sum_{\omega \in \Omega_{2}}\left(M_{r}\left(t_{p}, x^{(l)}, \omega, k, g\right)-M\left(t_{p-1}, \omega, k\right)\right)\right|<\left|\sum_{\omega \in \Omega_{1}}\left(M\left(t_{p}, x^{(s)}, \omega, k, g\right)-M\left(t_{p-1}, \omega, k\right)\right)\right|
$$

or

$$
\sum_{\omega \in \Omega_{2}}\left(M_{r}\left(t_{p}, x^{(l)}, \omega, k, g\right)-M\left(t_{p-1}, \omega, k\right)\right)=0,
$$

then the socioeconomic resources in regions $\omega \in \Omega_{2}$ are insufficient (if any) for the solution of the demographic problem in the domain $\Omega_{1}$ (under the condition that risk is absent). The problem is reduced to the problem of distribution of the available resources from regions $\omega \in \Omega_{2}$ among regions $\omega \in \Omega_{1}$. In this case, the situation of redistribution of the demographic resources between regions $\omega \in \Omega_{1}$ can arise. Therefore, we take a subset of regions $\Omega_{1}^{+}\left(\Omega_{1}=\Omega_{1}^{-} \cup \Omega_{1}^{+}, \Omega_{1}^{-} \cap \Omega_{1}^{+}=\varnothing\right)$ that can be demographic "donors" for the subset of regions $\Omega_{1}^{-}$from the ordered set of $\omega \in \Omega_{1}$. Then, for all $\omega^{*} \in \Omega_{1}^{+}$, we have

$$
M\left(t_{p}, x^{(s)}, \omega^{*}, k, g\right) \sum_{\omega \in \Omega_{1}}\left(M\left(t_{p}, x^{(s)}, \omega, k, g\right)-M\left(t_{p-1}, \omega, k\right)\right) \leq 0 .
$$

Using the procedures of items 4-6, determine the values of control parameters for which the trajectories at a moment of time $t_{p}$ take values in the set $\left[x_{l}\left(t_{p-1}, \omega, k, g_{l p}\right), x_{l}^{(s)}\left(t_{p}, \omega, k, g_{l p}\right)\right], \omega \in \Omega_{1}^{-}, l=\overline{1, n}$, that minimize the residuals

$$
\left(D_{r}\left(t_{p}, \omega, k\right)-D\left(t_{p}, x, \omega, k, g\right)\right)^{2} \leq \varepsilon_{1}\left\|\widetilde{D}\left(T_{0}\right)\right\|_{C\left(\Omega \times K_{0}\right)}^{2}
$$

and, at the same time, satisfy the migration balance equation

$$
\sum_{\omega \in \Omega} M(u(t, x, \omega, k, g))=0 .
$$

In regions $\omega \in \Omega_{1}^{+}$, the values of control parameters remain unchanged. For regions $\omega \in \Omega_{2}$, the set $\widetilde{G}$ in problem (22) consists of one collection of maximally admissible control parameters $\left\{g_{p}^{(l)}\right\}$ (the entire resource of regions is used for the 
solution of optimization problem (21)). This collection is assumed to be optimal. Go to item 11 .

10. If we have

$$
\begin{aligned}
& \left|\sum_{\omega \in \Omega_{2}}\left(M_{r}\left(t_{p}, x^{(l)}, \omega, k, g\right)-M\left(t_{p-1}, \omega, k\right)\right)\right| \\
\geq & \left|\sum_{\omega \in \Omega_{1}}\left(M\left(t_{p}, x^{(s)}, \omega, k, g\right)-M\left(t_{p-1}, \omega, k\right)\right)\right|, \\
& \sum_{\omega \in \Omega_{2}}\left(M_{r}\left(t_{p}, x^{(l)}, \omega, k, g\right)-M\left(t_{p-1}, \omega, k\right)\right) \neq 0,
\end{aligned}
$$

then regions $\omega \in \Omega_{2}$ contain sufficient socioeconomic resources for the solution of demographic problems in regions $\omega \in \Omega_{1}$. Based on the migration balance equation at moments of times $t_{p-1}$ and $t_{p}$, write an additional condition of equiproportional load connected with extraction of demographic resources in each region $\omega \in \Omega_{2}$. Search for the migration balance in regions $\omega \in \Omega_{2}$ in the form

$$
M\left(t_{p}, \omega, k\right)=M\left(t_{p-1}, \omega, k\right)+\beta\left(M_{r}\left(t_{p}, \omega, k\right)-M\left(t_{p-1}, \omega, k\right)\right),
$$

where $\beta$ is a scalar parameter determined from the migration balance equation,

$$
\beta=-\frac{\sum_{\omega \in \Omega_{1}}\left(M\left(t_{p}, x^{(s)}, \omega, k, g\right)-M\left(t_{p-1}, \omega, k\right)\right)}{\sum_{\omega \in \Omega_{2}}\left(M_{r}\left(t_{p}, x^{(l)}, \omega, k, g\right)-M\left(t_{p-1}, \omega, k\right)\right)} .
$$

By virtue of condition (17), we have $0<\beta \leq 1$. The migration balance and the corresponding population size for regions $\omega \in \Omega_{2}$ are as follows:

$$
\begin{gathered}
M\left(t_{p}, \omega, k\right)=M\left(t_{p-1}, \omega, k\right)-\frac{M_{r}\left(t_{p}, x^{(l)}, \omega, k, g\right)-M\left(t_{p-1}, \omega, k\right)}{\sum_{\omega \in \Omega_{2}}\left(M_{r}\left(t_{p}, x^{(l)}, \omega, k, g\right)-M\left(t_{p-1}, \omega, k\right)\right)} \Delta M_{\Omega_{1}}, \\
D_{r}\left(t_{p}, \omega, k\right)=D\left(M\left(t_{p}, \omega, k\right)\right) .
\end{gathered}
$$

Using the procedures of items $4-6$, determine the values of control parameters that minimize the residuals for regions $\omega \in \Omega_{2}$,

$$
\left(D_{r}\left(t_{p}, \omega, k\right)-D\left(t_{p}, x, \omega, k, g\right)\right)^{2} \leq \varepsilon_{1}\left\|\widetilde{D}\left(T_{0}\right)\right\|_{C\left(\Omega \times K_{0}\right)}^{2}
$$

and go to item 11 .

11. Check the condition of proximity of the value of the functional $J_{1}$ to zero with a prescribed accuracy or the attainment of a final moment of time,

$$
J_{1}\left(t_{p}, k, g\right)=\sum_{\omega \in \Omega_{1}} \lambda_{1}(\omega)\left[\widetilde{D}\left(T_{0}, \omega, k\right)-D\left(t_{p}, x, \omega, k, g\right)\right]^{2} \leq \varepsilon_{1}\left\|\widetilde{D}\left(T_{0}\right)\right\|_{C\left(\Omega \times K_{0}\right)}^{2} \text { or } p=P_{0} .
$$

If condition (18) is true, then output the result and go to item 12; otherwise, go to item 2.

12. Repeate this algorithm for all the social groups $k \in K_{0}$.

\section{SCENARIO OF OPTIMAL CONTROL WITH RISK MINIMIZATION}

For problem (1), we consider the case when $A=E$ is a unit operator. Additional constraints on the scenario are formulated in the form

$$
\widetilde{g}=\operatorname{Arg} \min _{g \in G}\left(J_{1}\left(T_{0}, k, g\right)\right), \quad \widetilde{g} \in \widetilde{G},
$$




$$
\begin{gathered}
g^{*}=\underset{g \in \widetilde{G}}{\operatorname{Arg} \min _{\tilde{J}}}\left(\widetilde{J}_{2}\left(T_{0}, k, g\right)\right), g^{*} \in G^{*}, \\
\widetilde{J}_{2}\left(T_{0}, k, g\right)=\sum_{\omega \in \Omega \backslash \Omega_{1}} \lambda_{2}(\omega) r\left(T_{0}, \omega, k, g\right) .
\end{gathered}
$$

Condition (19) requires the minimization of deviations of the population size from the values specified by the Center on the territory $\Omega_{1}$, and restriction (20) requires the risk minimization in the other part of the territory of the domain $\Omega_{2}$ for admissible optimal controls obtained in $\Omega_{1}$. Taking into account the nonnegativity of the functionals $J_{1}$ and $J_{2}$, conditions (19) and (20) mean that the Center $C_{0}$ considers the scenario of development of the migration process when, in problem (1), the desirable distribution of social groups among given regions of the domain $\Omega_{1}$ is more preferable (the functional $J_{1}$ ) than the absence of demographic risk in this territory (the functional $J_{2}$ ), i.e., the weight coefficient $\lambda_{1}(\omega)>>\lambda_{2}(\omega), \omega \in \Omega_{1}$. One should also take into account that we have $r_{0}(\omega, k) \geq 0$. Since controls are considered over the class of piecewise-constant bounded functions (the compactness of the set $G$ ) [1] and the operator $A$ is continuous in $R^{n}$ (1), condition (19) determines a compact set of optimal controls $g \in \widetilde{G} \subset G$. Problem (1), (19), (20) is correctly stated. Speed problem (1), (19), (20) can also be formulated in terms of discrete optimization theory [9]. Considering the functional of population size (5), in each segment $\left[t_{p-1}, t_{p}\right]\left(p=\overline{1, P_{0}}\right)$, we determine a collection of control parameters $g \in \widetilde{G} \subset G$ that provide the minimum of the following functionals:

$$
\begin{aligned}
& \widetilde{g}_{p}(\omega, k)=\left(\widetilde{g}_{1 p}(\omega, k), \ldots, \widetilde{g}_{n p}(\omega, k)\right)=\arg \inf _{g_{p} \in G}\left(J_{1}\left(t_{p}, k, g_{p}\right)\right), \\
& g_{p}^{*}(\omega, k)=\left(g_{1 p}^{*}(\omega, k), \ldots, g_{n p}^{*}(\omega, k)\right)=\arg \inf _{g_{p} \in G}\left(\widetilde{J}_{2}\left(t_{p}, k, g_{p}\right)\right)
\end{aligned}
$$

sequentially for each $p=1,2, \ldots$. Computations come to an end after the attainment of the minimum of functionals (21) and (22) with a preassigned error $\varepsilon>0$ when $p=p^{*}$ or after the attainment of a final moment of time when $p=P_{0}$. The gradient of the functional $J_{1}$ for the domain $\Omega_{1}$ is determined by formula (7). The gradient of the functional $J_{2}$ for $\Omega_{2}$ is similarly determined by formula (8) in the fixed segment $\left[t_{p-1}, t_{p}\right]$ as follows:

$$
\begin{gathered}
\frac{\partial J_{2}\left(t_{p}, k, g,\right)}{\partial g_{l^{*} p}}=\sum_{\omega \in \Omega \backslash \Omega_{1}} \frac{2 \lambda_{2}(\omega)}{\left(t_{p}-t_{0}\right)} \int_{t_{p-1}}^{t_{p}} \frac{\partial D(\tau, x, \omega, k, g)}{\partial g_{l^{*} p}} R(\tau, x, \omega, k, g) d \tau, \\
R(\tau, x, \omega, k, g)=(D(M(\tau, x, \omega, k, g))-\breve{D}(\tau, \omega, k)) \chi(\breve{D}(\tau, \omega, k)-D(M(\tau, x, \omega, k, g))) \\
+(D(M(\tau, \omega, k, g))-\widehat{D}(\tau, \omega, k)) \chi(D(M(\tau, \omega, k, g))-\widehat{D}(\tau, \omega, k)) . .
\end{gathered}
$$

Formulas (23) and (8) implies that the sign of the $l^{*}$-component of the gradient of the functional $J_{2}$ is determined by the sign of $(D(M(\tau, x, \omega, k, g))-\breve{D})$ or $(D(M(\tau, \omega, k, g))-\widehat{D}(\tau, \omega, k))$ and the sign of the parameter $\gamma_{l^{*}}(k)$. In fact, this means that optimal values of the $l^{*}$-component of the system trajectory $x_{l^{*}}\left(t_{p}\right)$ are in the segment connecting the initial value of the system trajectory with the projection of a given point onto the boundary of the compact domain determined by the constraints of problem (1). The properties of the gradients of the functionals $J_{1}\left(t_{p}, k, g\right)$ and $J_{2}\left(t_{p}, k, g\right)$ and the property of smoothness of the function of the population size $D\left(t_{p}, x, \omega, k, g\right)$ as a function of control parameters imply a numerical algorithm of solution of problem (1), (19), (20). We fix $k \in K_{0}$ (the $C_{k}$ th social group).

1. Specify the initial parameters of the problem being solved and characteristic constants of problem (1).

2. Specify the current step with respect to time $p \geq 1$ and determine the moment of time $t_{p}=t_{p-1}+\tau_{0}$.

3. Fix the sequent region $\omega \in \Omega_{1}$. By formulas (7) and (8), determine the initial value of the auxiliary control parameter $\Delta_{l p}(\omega, k), l=\overline{1, n}$, as follows: 


$$
\Delta_{l p}(\omega, k)= \begin{cases}\widehat{L}_{l p}(\omega, k) / N_{\gamma} & \text { if } \quad\left(\gamma_{l}(k)\left(D\left(t_{p-1}, x, \omega, k, g\right)-\widetilde{D}\left(t_{p}, \omega, k\right)\right)\right)<0, \\ \breve{L}_{l p}(\omega, k) / N_{\gamma} & \text { if } \quad\left(\gamma_{l}(k)\left(D\left(t_{p-1}, x, \omega, k, g\right)-\widetilde{D}\left(t_{p}, \omega, k\right)\right)\right)>0, \\ 0 & \text { if } \quad\left(\gamma_{l}(k)\left(D\left(t_{p-1}, x, \omega, k, g\right)-\widetilde{D}\left(t_{p}, \omega, k\right)\right)\right)=0 .\end{cases}
$$

Here, $N_{\gamma}$ is the initial value of the number of steps along the segment connecting the initial point of the system trajectory with the boundary point determined by the boundary value of a compact set $G \subset R^{n}$. It follows from formulas (5), (7), and (8) that the maximal value of the population size $D_{\max }\left(t_{p}, \omega\right)$ is attained during the motion of the system in the direction of increasing the values of stimulators $g_{l p}(\omega, k)=\widehat{L}_{l p}(\omega, k)$ (when $\gamma_{l}>0$ ) to their maximum and decreasing the values of destimulators of the social system $g_{l p}(\omega, k)=\breve{L}_{l p}(\omega, k)$ (when $\gamma_{l}<0$ ) to their minimum. The minimal value of the population size $D_{\min }\left(t_{p}, \omega\right)$ is similarly attained but in the other direction. It is obvious that the population size assumes the value that minimizes the functional $J_{1}$ in the internal domain of the set $\widetilde{G}$ if we have $D_{\min }\left(t_{p}, \omega\right)<\widetilde{D}\left(t_{p}, \omega\right)<D_{\max }\left(t_{p}, \omega\right)$ or on the boundary of the set $\widetilde{G}$ if we have $\widetilde{D}\left(t_{p}, \omega\right)>D_{\max }\left(t_{p}, \omega\right)$ or $\widetilde{D}\left(t_{p}, \omega\right)<D_{\min }\left(t_{p}, \omega\right)$. In the latter case, optimal values of control parameters are on the boundary of the domain: $g_{l p}^{*}(\omega, k)=\widehat{L}_{l p}(\omega, k)\left(\right.$ when $\left.\gamma_{l}>0\right), g_{l p}^{*}(\omega, k)=\breve{L}_{l p}(\omega, k)\left(\right.$ when $\left.\gamma_{l}<0\right)$ or $g_{l p}^{*}(\omega, k)=\breve{L}_{l p}(\omega, k)\left(\right.$ when $\left.\gamma_{l}>0\right)$, and $g_{l p}^{*}(\omega, k)=\widehat{L}_{l p}(\omega, k)$ (when $\left.\gamma_{l}<0\right)$. It is also obvious that the optimal control in the internal domain $\widetilde{G}$ can be not unique. Then the unique solution is chosen with the help of additional requirements on the minimization of the computational complexity of the algorithm, for example, by the construction of a minimizing sequence by formulas (24) and (10).

4. By formulas (9) and (10), compute the values $(s)$ of the control parameter $g_{l p}^{(s)}(\omega, k)$ and the coordinates of the (s)-element of the minimizing sequence, i.e., the system trajectory $x_{l}^{(s)}\left(t_{p}, \omega, k, g_{l p}\right), \omega \in \Omega_{1}, l=\overline{1, n}(s \geq 1)$. Compute the values of the functions $u\left(t_{p}, x^{(s)}, \omega, k\right), M\left(u\left(t_{p}, x^{(s)}, \omega, k, g\right)\right)$, and $D\left(t_{p}, x^{(s)}, \omega, k, g\right)$.

5. Check the condition of minimum of residual (12) with a prescribed error $\varepsilon_{1}$. If condition (12) is true, then go to item 6.

Otherwise, check the conditions of monotony of residual (13), (13a). If conditions (13), (13a) are true, then increase $s$ by unity, assume that $\alpha^{(s)}=\alpha^{(s-1)}$, and go to the beginning of item 4 to compute the system trajectory at the new step of iterations. Otherwise, reduce the value of $\alpha^{(s)}$ by a factor of two, check the condition $\alpha^{(s)}<\varepsilon_{2}$ of attainability of the limit of resources in this region. If this condition is true, go to item 6 and, otherwise, go to the beginning of item 4 and again compute the $(s)$-values of the control parameter and system trajectory.

6. If the set $\Omega_{1}$ is not exhausted, then go to item 3 and, otherwise, go to item 7 .

7. For each region $\omega \in \Omega_{2}$, determine maximally admissible values of control parameters $g_{l p}^{(l)}(\omega, k)$ by formulas (14) and (15). Since the migration balance equation has been true at the previous moment of time $t_{p-1}$, at the current step, based on the analysis of the change in the direction of the migration process (obtained as result of solution of the optimization problem for the domain $\Omega_{1}$ ), determine the greatest possible socioeconomic resources of the domain $\Omega_{2}$ that are necessary for compensation of the arising disbalance. For greatest possible controls (14) and (15), compute the system trajectory $x_{l}^{(l)}\left(t_{p}, \omega, k, g_{l p}\right)$ by formula (11) and the values of the functions $u\left(t_{p}, x^{(l)}, \omega, k\right), M\left(U\left(t_{p}, x^{(l)}, \omega, k, g\right)\right)$, and $D\left(t_{p}, x^{(l)}, \omega, k, g\right)$. By virtue of formula (15), the following inequality is true:

$$
\sum_{\omega \in \Omega_{2}}\left(M\left(t_{p}, x^{(l)}, \omega, k, g\right)-M\left(t_{p-1}, \omega, k\right)\right) \times \sum_{\omega \in \Omega_{1}}\left(M\left(t_{p}, x^{(s)}, \omega, k, g\right)-M\left(t_{p-1}, \omega, k\right)\right) \leq 0 .
$$

8. If the following inequlity is true:

$$
\left|\sum_{\omega \in \Omega_{2}}\left(M\left(t_{p}, x^{(l)}, \omega, k, g\right)-M\left(t_{p-1}, \omega, k\right)\right)\right|<\left|\sum_{\omega \in \Omega_{1}}\left(M\left(t_{p}, x^{(s)}, \omega, k, g\right)-M\left(t_{p-1}, \omega, k\right)\right)\right|,
$$


then the socioeconomic resources in the regions $\omega \in \Omega_{2}$ are insufficient (if any) for the solution of the demographic problem in the domain $\Omega_{1}$. The problem is similar to that of item 9 from the previous scenario. Take a subset of regions $\Omega_{1}^{+}\left(\Omega_{1}=\Omega_{1}^{-} \cup \Omega_{1}^{+}, \Omega_{1}^{-} \cap \Omega_{1}^{+}=\varnothing\right)$ that can be "donors" for the subset of regions $\Omega_{1}^{-}$from the ordered set of $\omega \in \Omega_{1}$, i.e., for all $\omega^{*} \in \Omega_{1}^{+}$, the following inequality is true:

$$
M\left(t_{p}, x^{(s)}, \omega^{*}, k, g\right) \sum_{\omega \in \Omega_{1}}\left(M\left(t_{p}, x^{(s)}, \omega, k, g\right)-M\left(t_{p-1}, \omega, k\right)\right) \leq 0 .
$$

Using the procedures of items 4-6, determine the values of control parameters for which the trajectories at the moment of time $t_{p}$ assume values from the set $\left[x_{l}\left(t_{p-1}, \omega, k, g_{l p}\right), x_{l}^{(s)}\left(t_{p}, \omega, k, g_{l p}\right)\right], \omega \in \Omega_{1}^{-}, l=\overline{1, n}$, that minimize the residuals

$$
\left(D_{r}\left(t_{p}, \omega, k\right)-D\left(t_{p}, x, \omega, k, g\right)\right)^{2} \leq \varepsilon_{1}\left\|\widetilde{D}\left(T_{0}\right)\right\|_{C\left(\Omega \times K_{0}\right)}^{2}
$$

and simultaneously satisfy the migration balance equation

$$
\sum_{\omega \in \Omega} M(u(t, x, \omega, k, g))=0 .
$$

The values of control parameters in regions $\omega \in \Omega_{1}^{+}$remain unchanged. For regions $\omega \in \Omega_{2}$ in problem (22), the set $\widetilde{G}$ consists of one collection of maximally admissible control parameters $\left\{g_{p}\right\}$ (all the resources of these regions are spent for the solution of optimization problem (21)). It is this collection that is assumed to be optimal. Go to item 10 .

9. If the following inequality is true:

$$
\begin{aligned}
& \left|\sum_{\omega \in \Omega_{2}}\left(M\left(t_{p}, x^{(l)}, \omega, k, g\right)-M\left(t_{p-1}, \omega, k\right)\right)\right| \\
& \geq\left|\sum_{\omega \in \Omega_{1}}\left(M\left(t_{p}, x^{(s)}, \omega, k, g\right)-M\left(t_{p-1}, \omega, k\right)\right)\right|,
\end{aligned}
$$

then regions $\omega \in \Omega_{2}$ contain sufficient socioeconomic resources for the solution of demographic problems in regions $\omega \in \Omega_{1}$. Using the procedures of items 4-6, determine the values of control parameters for which the trajectories at the moment of time $t_{p}$ assume values from the set $\left[x_{l}\left(t_{p-1}, \omega, k, g_{l p}\right), x_{l}^{(l)}\left(t_{p}, \omega, k, g_{l p}\right)\right], \omega \in \Omega_{2}, l=\overline{1, n}$, that minimize the risk

$$
\begin{gathered}
R^{2}(\tau, x, \omega, k, g)=((D(M(\tau, x, \omega, k, g))-\breve{D}(\tau, \omega, k)) \chi(\breve{D}(\tau, \omega, k)-D(M(\tau, x, \omega, k, g))) \\
+(D(M(\tau, x, \omega, k, g))-\widehat{D}(\tau, \omega, k)) \chi(D(M(\tau, x, \omega, k, g))-\widehat{D}(\tau, \omega, k)))^{2} \leq \varepsilon_{1}\left\|\widetilde{D}\left(T_{0}\right)\right\|_{C\left(\Omega \times K_{0}\right)}^{2}
\end{gathered}
$$

and simultaneously satisfy the migration balance equation

$$
\sum_{\omega \in \Omega} M(u(\tau, x, \omega, k, g))=0 .
$$

10. Check the following condition of proximity of the functional $J_{1}$ to zero with a prescribed accuracy or the attainment of the final moment of time:

$$
J_{1}\left(t_{p}, k, g\right)=\sum_{\omega \in \Omega_{1}} \lambda_{1}(\omega)\left[\widetilde{D}\left(T_{0}, \omega, k\right)-D\left(\tau_{p}, x, \omega, k, g\right)\right]^{2} \leq \varepsilon_{1}\left\|\widetilde{D}\left(T_{0}\right)\right\|_{C\left(\Omega \times K_{0}\right)}^{2} \text { or } p=P_{0} .
$$

If condition (26) is true, then output the result and go to item 11; otherwise, go to item 2.

11. Repeat this algorithm for all social groups $k \in K_{0}$. 
TABLE 1. Optimal Model Data on the Population Size in the Oblasts of Ukraine according to the Scenario of the Transregional Migration with Zero Risk

\begin{tabular}{|c|c|c|c|c|c|}
\hline Oblast & $D_{0}$ & D & $\hat{D}$ & $D_{\text {opt }}$ & $\tilde{D}$ \\
\hline$\Omega_{1}$ & & & & & \\
\hline Volynsk oblast & 1061.1 & 900 & 1200 & 1082.27 & 1090 \\
\hline $\begin{array}{c}\text { Chernovtsy oblast } \\
\Omega_{2}\end{array}$ & 938.2 & 800 & 1050 & 956.40 & 970 \\
\hline Vinnitsa oblast & 1819.7 & 1700 & 1900 & 1803.25 & - \\
\hline Dnepropetrovsk oblast & 3733.6 & 3600 & 3800 & 3701.81 & - \\
\hline Donetsk oblast & 4987.3 & 4800 & 5100 & 4934.77 & - \\
\hline Zhitomir oblast & 1437.7 & 1300 & 1500 & 1425.16 & - \\
\hline Zakarpatye oblast & 1280.7 & 1200 & 1350 & 1277.84 & - \\
\hline Zaporozhye oblast & 2016.0 & 1900 & 2100 & 1999.47 & - \\
\hline Ivano-Frankovsk oblast & 1450.3 & 1300 & 1550 & 1445.69 & - \\
\hline Kiev oblast & 1844.0 & 1700 & 2000 & 1824.00 & - \\
\hline Kirovograd oblast & 1172.5 & 1050 & 1300 & 1157.54 & - \\
\hline Lugansk oblast & 2668.1 & 2500 & 2800 & 2635.05 & - \\
\hline Lvov oblast & 2708.3 & 2600 & 2850 & 2696.80 & - \\
\hline Nikolaev oblast & 1307.6 & 1200 & 1400 & 1297.48 & - \\
\hline Odessa oblast & 2510.2 & 2400 & 2650 & 2492.57 & - \\
\hline Poltava oblast & 1688.8 & 1550 & 1800 & 1672.10 & - \\
\hline Rovno oblast & 1184.9 & 1050 & 1300 & 1183.16 & - \\
\hline Sumy oblast & 1349.3 & 1250 & 1450 & 1333.47 & - \\
\hline Ternopol oblast & 1159.0 & 1050 & 1300 & 1152.46 & - \\
\hline Kharkov oblast & 2977.6 & 2800 & 3100 & 2950.80 & - \\
\hline Kherson oblast & 1234.1 & 1100 & 1350 & 1225.13 & - \\
\hline Khmelnytskii oblast & 1468.4 & 1350 & 1600 & 1455.51 & - \\
\hline Cherkassy oblast & 1459.5 & 1350 & 1600 & 1446.76 & - \\
\hline Chernigov oblast & 1299.8 & 1200 & 1400 & 1281.90 & - \\
\hline Kiev & 2596.0 & 2400 & 2800 & 2602.36 & - \\
\hline Sevastopol & 391.9 & 320 & 450 & 389.69 & - \\
\hline $\begin{array}{l}\text { Autonomous Republic } \\
\text { Crimea }\end{array}$ & 2106.3 & 2000 & 2150 & 2094.37 & - \\
\hline
\end{tabular}

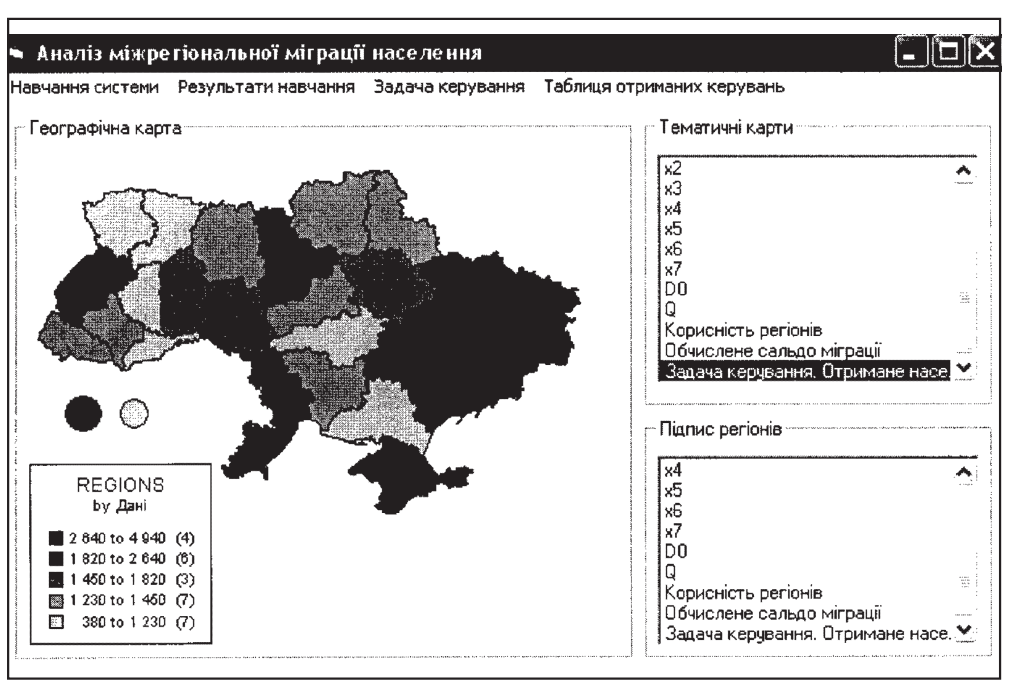

Fig. 1

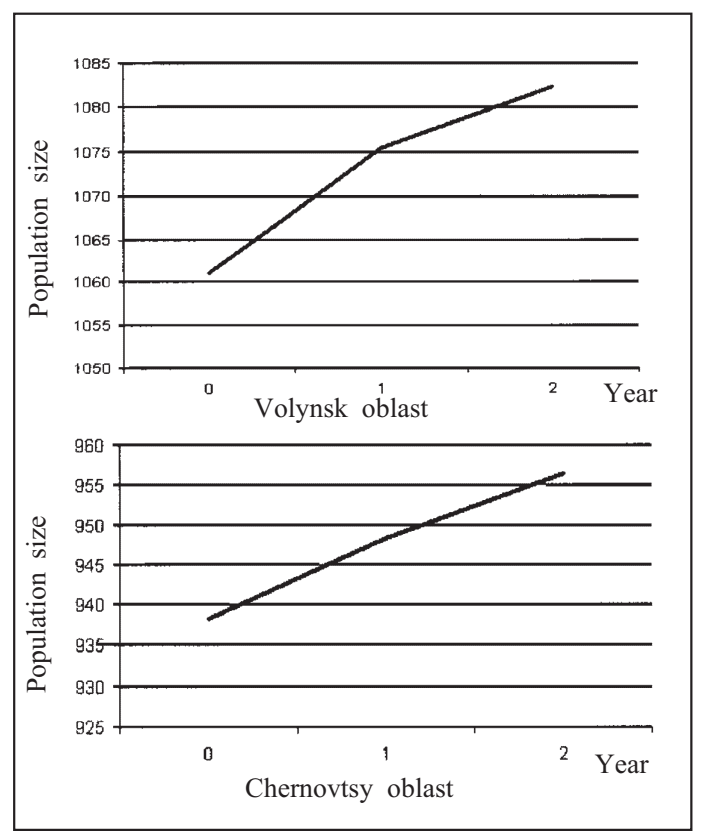

Fig. 2 
Based on the developed models and algorithms, an applied geoinformation system (GIS) is created that supports making decisions on the management of transregional migration processes on the territory of Ukraine [10-12]. For problem (1), the territory of Ukraine with 25 oblasts and two special centers of population (Kiev and Sevastopol), $\Omega=27$, and one social group of "statistical average citizens," $K_{0}=1$, are considered. The basic control parameters $j_{0}=7$ and the parameter $q=3$ were used. Calculations were based on data from [13, 14] for Ukraine over 1999-2004.

The results of calculations for the scenarios of optimal control of transregional migration with zero risk at a final moment of time are presented in Table 1. Figure 1 presents the map of the population size in the Ukrainian regions after the solution of the control problem according to the scenario with zero risk, and Fig. 2 presents the diagrams of changing the population size in the regions of $\Omega_{1}$ for the first scenario (according to the results of functioning of the GIS).

For the considered variant of problem (1), the number of variables used for the solution of extremal problems equals $N_{\text {tot }}=\Omega \times j_{0}=189$ for each fixed time interval. Therefore, the use of the developed algorithms of directed search for optimal solutions allowed us to considerably decrease the time of numerical solution in comparison with algorithms of exhaustive search over the global set and to solve this problem in real time.

\section{REFERENCES}

1. V. V. Akimenko and O. G. Nakonechnyi, "Optimal control models for interregional migration under social risks," Cybernetics and Systems Analysis, No 3, 107-122 (2006).

2. N. N. Moiseyev, Mathematical Problems of Systems Analysis [in Russian], Nauka, Moscow (1986).

3. M. D. Mesarovich et al., Theory of Hierarchical Multilevel Systems [Russian translation], Mir, Moscow (1982).

4. V. V. Akimenko, "A computer system supporting administrative decision making under the conditions of mixed information for systems of ecological monitoring of the atmosphere," Cybernetics and Systems Analysis, No. 5, 151-167 (2000).

5. A. G. Nakonechnyi and V. P. Martsenyuk, "Controllability Problems for Differential Gompertzian Dynamic Equations," Cybernetics and Systems Analysis, No. 2, 123-133 (2004).

6. E. B. Lee and L. Markus, Foundations of Optimal Control Theory, Wiley, New York-London-Sydney (1967).

7. V. M. Alekseev, V. M. Tikhomirov, and S. V. Fomin, Optimal Control [in Russian], Nauka, Moscow (1979).

8. O. V. Staroverov and S. N. Kotelnikova, Modeling of Social and Economic Processes [in Russian], Mosk. Gos. Inst. Elektroniki i Matematiki (Tekhn. Univ.), Moscow (2001).

9. I. V. Sergienko and V. P. Shilo, Discrete Optimization Problems [in Russian], Naukova Dumka, Kiev (2003).

10. M. N. DeMers, Fundamentals of Geographic Information Systems, Wiley, New York (1997).

11. V. Akimenko and O. Nakonechnyi, "A speed model of optimal control of migration processes under integrated social risks," in: Proc. Conf. on Problems of Decision-Making under Uncertainty, Taras Shevchenko University, Kyiv-Berdyansk (2005), pp. 79-80.

12. V. V. Akimenko and O. G. Nakonechnyi, "Optimal control of transregional migration processes, taking into account social risks," in: Proc. Conf. on Demographic Development of Ukraine and Priority Tasks in Population Policy, KNEU, Kyiv (2005), pp. 45-48.

13. O. S. Vlasyuk and S. V. Pirozhkov, Human Development Index: Ukrainian Experience [in Ukrainian], PROON, Kiev (1995).

14. Statistical Year-Book of Ukraine for 2004 [in Ukrainian], Konsultant, Kiev (2004). 